\title{
BMJ Open A health economic model for the development and evaluation of innovations in aged care: an application to consumer-directed care-study protocol
}

\author{
Julie Ratcliffe, ${ }^{1}$ Emily Lancsar, ${ }^{2}$ Mary Luszcz, ${ }^{3}$ Maria Crotty, ${ }^{4}$ Len Gray, ${ }^{5}$ \\ Jan Paterson, ${ }^{6}$ Ian D Cameron ${ }^{7}$
}

To cite: Ratcliffe J, Lancsar $\mathrm{E}$, Luszcz $\mathrm{M}$, et al. A health economic model for the development and evaluation of innovations in aged care:

an application to consumerdirected care-study protocol. BMJ Open 2014;4 e005788. doi:10.1136/ bmjopen-2014-005788

- Prepublication history for this paper is available online. To view these files please visit the journal online (http://dx.doi.org/10.1136/ bmjopen-2014-005788)

Received 26 May 2014 Accepted 2 June 2014

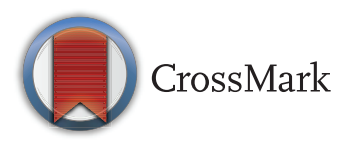

For numbered affiliations see end of article.

Correspondence to Professor Julie Ratcliffe; julie.ratcliffe@flinders.edu.au

\section{ABSTRACT}

Introduction: Consumer-directed care is currently being embraced within Australia and internationally as a means of promoting autonomy and choice in the delivery of health and aged care services. Despite its wide proliferation little research has been conducted to date to assess the views and preferences of older people for consumer-directed care or to assess the costs and benefits of such an approach relative to existing models of service delivery.

Methods and analysis: A comprehensive health economic model will be developed and applied to the evolution, implementation and evaluation of consumer-directed care in an Australian community aged care setting. A mixed methods approach comprising qualitative interviews and a discrete choice experiment will determine the attitudes and preferences of older people and their informal carers for consumer-directed care. The results of the qualitative interviews and the discrete choice experiment will inform the introduction of a new consumer-directed care innovation in service delivery. The cost-effectiveness of consumer-directed care will be evaluated by comparing incremental changes in resource use, costs and health and quality of life outcomes relative to traditional services. The discrete choice experiment will be repeated at the end of the implementation period to determine the extent to which attitudes and preferences change as a consequence of experience of consumer-directed care. The proposed framework will have wide applicability in the future development and economic evaluation of new innovations across the health and aged care sectors.

Ethics and dissemination: The study is approved by Flinders University Social and Behavioural Research Ethics Committee (Project No. 6114/SBREC). Findings from the qualitative interviews, discrete choice experiments and the economic evaluation will be reported at a workshop of stakeholders to be held in 2015 and will be documented in reports and in peer reviewed journal articles.

\section{INTRODUCTION}

Australia's population is ageing rapidly and by 2045 it is estimated that 1 in 4 Australians will be aged 65 years or more and nearly 1 in 10 will be 80 years or over. ${ }^{1}$ This demographic transformation poses major challenges for public policy as many indicators suggest that ageing will exert substantial upward pressure on aged care expenditure in the coming decades. ${ }^{2}$ In addition, factors other than an ageing population, including increasing affluence coupled with a generational desire for greater autonomy and choice, are creating new pressures and challenges for the aged care sector. It is government policy and the wish of the vast majority of older people to remain, and be cared for, in their own homes for as long as possible. ${ }^{3}$ The advantages, for older people and their families, of remaining in the community are significant and include less disruption and more continuity of care in a familiar environment. ${ }^{3}$ Current trends in the provision of aged care services show that older people are exhibiting an increased preference for independent living arrangements supported by community care. ${ }^{1}$

Consumer-directed care (CDC) is currently being embraced within Australia and internationally as a means to promote autonomy and choice and to provide improved outcomes to facilitate ageing in place for older people in a community setting. ${ }^{4-7}$ While there is no single definition of CDC, and several variations of CDC have been identified in the literature, the distinguishing feature of CDC concerns the service recipients or their representatives (where personal cognitive capacity is limited) having 'control' 
over funds allocated, so they can be used preferentially to meet the individual's needs. It thus represents the opposite of provider-directed care (PDC) which is controlled entirely by the service provider. ${ }^{8}$ The Australian Department of Health and Ageing recently introduced reforms to the aged care sector which indicate that all new publicly funded community care packages, designed to assist people aged over 65 years of age remain living at home for as long as possible, must be offered on a CDC basis by service providers. ${ }^{3}$ Under this scheme the provider remains as the fund-holder, but expends each individual's budget as directed by the older person. This Self-Directed Care option focuses on increasing recipients' control over care arrangements in order to increase flexibility and provide programmes and services which better meet the needs of the older person. In contrast Cash-for-Care schemes, typically involve untied provision of cash payments or vouchers to enable older people to manage their own funds and purchase their own care directly. ${ }^{9}$ Self-directed care has a potential advantage in that it reduces the burden of management of CDC for the older person since responsibility for budgeting arrangements and the management of funds remains with the service provider. ${ }^{6} 910$

Previous findings from a large scale evaluation of the individual budgets pilot programme in the $\mathrm{UK}^{6}$ raised concerns about the benefits of CDC for older people and how these can be maximised. For some older people, the anxiety and stress associated with potential changes to established support arrangements moderated any potential gains from the increased transparency, control and flexibility offered by CDC. It was concluded that the benefits of CDC may take some time to be realised in practice since it may take some time for older people to develop the confidence to assume greater control. ${ }^{6}$ Translations of these findings into the Australian context are hampered by differences in the administrative, sociopolitical and legislative contexts of the programmes across countries.

To date, very few studies have been undertaken internationally to assess the views and preferences of older people and their informal carers regarding the basic features which should make up a CDC approach to community aged care. Information relating to the cost-effectiveness of CDC in an Australian community aged care setting is also notably absent and will therefore be addressed by this study.

\section{Objective}

This paper describes the study protocol to apply a health economic framework to the development and evaluation of a new consumer directed care approach to community aged care service delivery. The specific aims of the study are to:

1. Determine the attitudes and preferences of older people and their informal carers for a CDC approach.
2. Assess the extent to which attitudes and preferences change over time as a consequence of exposure to CDC.

3. Investigate incremental changes in health, quality of life and psychological well-being for older people and their informal carers as a consequence of exposure to CDC.

4. Determine the cost-effectiveness of the new CDC approach to community aged care service delivery.

\section{METHODS AND ANALYSIS}

A comprehensive health economic model will be applied, adopting both qualitative and quantitative approaches including cost-effectiveness analysis and discrete choice experiments (DCEs) for investigating older people and informal carer preferences. ${ }^{11}$ DCEs are an attribute-based measure of the benefits of services or programmes based on two main assumptions. First, that health and/or aged care services can be described or defined by their key characteristics (or attributes). Second, an individual's valuation or preference for alternative service configurations is dependent on the levels of these characteristics. ${ }^{12}$ DCE's were first introduced into health economics in recognition of the fact that consumers are typically concerned with many aspects of service delivery beyond health outcomes. ${ }^{13}{ }^{14}$ Such factors may include, for example, location in which care is provided, the type of care available and the personnel providing care. DCE's allow investigation of the tradeoffs individuals are prepared to make between such process attributes and/or between process and health outcomes attributes. The study will comprise four distinct phases to be implemented sequentially.

\section{Phase 1: Investigation of preintervention attitudes and preferences}

1. Qualitative interviews

A series of in-depth qualitative interviews will be conducted initially with 60 participants $(n=20$ older people, $\mathrm{n}=20$ informal carers and $\mathrm{n}=20$ aged care staff) who consent to be interviewed to determine knowledge of, and attitudes towards, CDC. The interviews will probe factors and issues relating to choice and control including: What do the concepts of choice and control mean to older people, their informal carers and aged care staff? How is control different from choice? What do older people, informal carers, aged care staff want from CDC in terms of control of the services received? What aspects of care do older people want to have control over? The interviews will also investigate the relationship between choice and control and the characteristics of the older person (eg, living alone vs with coresident, living with a family carer, people living with dementia and behaviour change, level of educational attainment) and how choice and control may impact on the lives of older people in relation to their confidence and self-esteem, family relationships and community and social participation. 
Data analysis: Interview transcripts will be entered into a computer-assisted qualitative data analysis programme, NVivo, for coding and analysis. A process of axial coding will be utilised where comparisons will be drawn between emerging themes. The final level of analysis will involve the abstraction of these themes into core categories and comparing these categories with existing theory on health, ageing, decision-making and aged care. ${ }^{15}$ The results of the qualitative analysis will be employed to inform the most appropriate attributes and levels for the DCE, thereby reflecting recommended best practice guidelines for the development of a DCE for the health and social welfare sectors. ${ }^{16}$ Candidate attributes for inclusion within the DCE may reflect, for example, the degree of involvement of the care coordinator in managing services and providing support, the portfolio of services for which CDC is applicable, the role and number of staff involved in providing services, provision of a facility to 'bank' unused hours of care for future use and alternative mechanisms for aligning the care needs deemed most important by older people with their assessed needs.

\section{Discrete choice experiment}

While there has been an exponential increase in the application of DCEs in healthcare in recent years, DCE studies within populations of older people remain relatively rare. ${ }^{11} 17$ The DCE will be developed for administration via a face-to-face interview with a sample of older people, or a proxy assessor (where cognitive impairment, defined as a score below 19 according to the Mini-Mental State Examination, precludes participation). The DCE will be piloted with a small number of older people $(n=10)$ to confirm attributes, question framing and develop consumer friendly wording.

Sample size for DCE's cannot be determined exactly in advance because one requires complete knowledge of the full factorial (ie, the number of attributes and levels to be included in the experiment). ${ }^{18}$ However, it has been demonstrated that large sample properties can be achieved with a minimum sample size of 50 respondents in each subgroup of interest. ${ }^{19}$ The DCE will be administered via a face-to-face interview with older people $(n=50)$ and informal carers $(n=50)$. The DCE will be based upon a fractional factorial design that permits the estimation of main effects, (while maintaining the design properties of orthogonality and level balancealso known as an orthogonal main effects plan). ${ }^{20}$ We propose to block the design into versions so that each participant is presented with no more than eight DCE choice questions. This will promote participant completion rates and minimise error due to fatigue.

Data analysis: Data from the DCE will be analysed using dichotomous choice models, for example, conditional or mixed logit regression. Mixed logit models allow for correlation of error components within participants, as well as correlation between alternatives where there is more than one alternative. Marginal rates of substitution (MRS) calculated as the ratio of attribute coefficients, will be used to assess the relative importance of the attributes and the trade-offs participants are willing to make between attributes for gains in another attribute. Preferences will be compared across discrete choice models (eg,older people vs informal carers vs staff) using two methods (1) correlations of the model coefficients and (2) by comparing the predicted MRS between attributes. ${ }^{18}$ Coefficients will be adjusted for scale using the Swait and Louviere ${ }^{19}$ approach allowing for scale adjusted comparison and the preference data from the subgroups will be tested to determine whether it can be pooled to estimate a joint model of preferences.

The findings from the qualitative interviews and the DCE study will be applied to inform the development of the features of the CDC model of service delivery to be implemented across the partner organisations.

\section{Phase 2: Introduction and development of the CDC model}

A new CDC service delivery approach will be developed and implemented in collaboration with the partner organisations. The findings from the qualitative interviews and the DCE study in phase 1 will be applied to identify and operationalise the features of the CDC service delivery approach to be implemented. The new approach will include access to a care coordinator provided by each of the partner organisations who will be responsible for providing opportunities for consumers and their informal carers to direct and manage their own care. The new CDC approach will be pretested with a small number of older people $(n=5)$. The principles of the new CDC model to be adopted will reflect the general policies governing the delivery of aged care services in Australia and will meet the requirements of the Australian Government's Department of Social Services that CDC in aged care should be integrated as an optional mode of care delivery operating within the constraints of the current legislative arrangements. The new CDC approach will provide opportunities for regular reviews to be undertaken in consultation with the care coordinator and the adjustment of budget and services according to the changing care needs of recipients and their informal carers.

\section{Phase 3: Economic evaluation}

Phase 3 will involve the implementation and economic evaluation of the new CDC approach identified and developed in phase 2 . The objectives of phase 3 will be to:

- Calculate the incremental changes in health, quality of life and well-being outcomes for older people and their informal carers in the CDC group compared to those receiving $\mathrm{PDC}$;

- Determine the cost-effectiveness of the new CDC approach relative to PDC.

Older people (or a proxy consenter where cognitive impairment precludes informed consent) and family caregivers will be invited to participate and provide informed consent. The comparator group will comprise 
consumers and their informal caregivers who are similar to the intervention group in terms of socioeconomic characteristics, cognitive impairment (dementia vs nondementia diagnosis) and living arrangements (living alone or with family/carer) but who opt to receive PDC.

A series of validated instruments will be applied, all of which are appropriate for interviewer administration. These instruments will be administered by face-to-face interview to both older people and their informal carers (with the exception of the Carer Experience Scale (CES) which will be administered to informal carers only) at baseline and at 6 month time points. A proxy assessor will be employed, where cognitive impairment in an older person precludes understanding and selfcompletion of the instruments.

1. ICECAP-O capability index for older people

The ICECAP-O index of capability for older people focuses on quality of life and includes five attributes: attachment, security, role, enjoyment and control. The index provides a rigorous approach to consider the value of different interventions for the purposes of economic evaluation within the aged care sector. ${ }^{21}$

\section{EuroQoL (EQ-5D)}

The EQ-5D is a generic preference-based measure of health and is one of the most widely used instruments for estimating quality-adjusted life years (QALYs) within the context of economic evaluation. The EQ-5D is simple to administer and has been found to have higher completion rates in older people relative to other generic preference-based measures of health. ${ }^{22}$

\section{Self-esteem scale}

The Self-esteem scale is a widely used self-esteem measure in social science research. The scale comprises 10 items with a Likert scale response ranging from almost always true to never true. The instrument has been widely applied by Professor Luszcz in the Australian Longitudinal Study of Ageing and other populations of older people. ${ }^{23} 24$

4. Pearlin Mastery Scale

The Pearlin Mastery Scale is seven-item scale with a likert scale response ranging from strongly agree to disagree. The instrument is a measure of self-concept and references the extent to which individuals perceive themselves in control of forces that significantly impact their lives. ${ }^{25}$

\section{CES}

The CES is a new instrument which has been designed to measure and value the quality of life of carers. The CES comprises six attributes of the caring experience (getting on, organisational assistance, social support, activities, control and fulfilment) and has been designed to provide a direct assessment of carers' welfare within an economic evaluation framework. ${ }^{26}$ All participants and their informal carers in the intervention and control groups will be monitored to assess changes in their health, quality of life and psychological well-being from recruitment until the end of the evaluation period.
Sample size and recruitment: A sample size of 64 in each group will have $80 \%$ power to detect a meaningful difference in health and quality of life state values or utilities of 0.05 between the two groups; assuming that the common $\mathrm{SD}$ is 0.100 using a two group t test with a 0.050 two-sided significance level. ${ }^{27}$ An average of six new clients per week from the four partner organisations will be recruited to the study over a period of 28 weeks, resulting in a total sample of 168 clients and their relevant family caregiver/s. Advice from our four partner organisations has indicated that this recruitment rate is feasible. Attrition may occur due to participants withdrawing from the project, admission to hospital or a nursing home or consumer death. An attrition rate of approximately $25 \%$ over the 6-month follow-up period (due to death and transfer to a residential aged care facility) has been allowed for, resulting in the full participation of 128 (64 intervention and 64 comparator participants).

Data analysis: The economic analysis will be conducted alongside the trial and will comprise a cost-effectiveness study of the CDC intervention versus usual care. The primary measures of outcome for the economic analysis will be cost per unit of improvement in quality of life as measured by the ICECAP and the cost per QALY as measured by the EQ-5D. Resource use and costs collected within the evaluation will include all elements associated with the provision of the proposed CDC programme versus usual care including establishment costs, the provision of a CDC navigator and financial management. The resource use and associated cost items will be measured within the trial by a series of semistructured telephone/face-to-face interviews with staff from each partner organisation involved in the administration of the CDC intervention and with clients and family carers from the intervention group. Mean costs and effectiveness between the intervention and control groups will be compared and incremental cost-effectiveness ratios (ICERs) presented in terms of the cost per (QALY) as measured by the EQ-5D. CIs will be presented around the ICERs and cost-effectiveness acceptability curves for varying threshold values of cost-effectiveness will also be presented. $^{28}$ An assessment of the sensitivity of the results obtained to variation in measured resource use, effectiveness and/or unit costs will be undertaken using appropriate one-way and multiway sensitivity analysis. ${ }^{29}$

\section{Phase 4: Investigation of postintervention attitudes and preferences}

The DCE study from phase 1 will be repeated at the end of the evaluation period to assess the extent to which the attitudes and preferences of older people and informal carers and staff have changed as a consequence of exposure to CDC. An assessment of the degree of convergence (or otherwise) over time between the attitudes and preferences of older people and their informal carers will be enabled by a comparison of the baseline and 6 months postintervention DCE study findings. 
Preferences will be compared across discrete choice models via an assessment of correlations of the model coefficients and a comparison of the predicted MRS between attributes.

\section{Ethics and dissemination}

Findings from the qualitative interviews, discrete choice experiments and the economic evaluation will be reported at a workshop of stakeholders to be held in 2015 , reports and in peer reviewed journals.

The outcomes of this study will include recommendations and knowledge about the attitudes and preferences of older people and their informal carers within Australia for a CDC approach to service delivery. The study will demonstrate how health economics methodologies can readily be applied in the development and economic evaluation of future innovations in service delivery across the health and aged care sectors. The proposed methodologies provide substantial impetus for the engagement of older people and their informal carers in the development of new approaches to service delivery. To our knowledge, the proposed study represents the first study internationally to develop and apply a comprehensive health economic model to the development, implementation and evaluation of service innovations in aged care. A unique component of the study is to undertake the DCE at two time-points (baseline and at the end of the evaluation period) to assess the extent to which the attitudes and preferences of older people and their informal carers and staff have changed as a consequence of experience of the new CDC approach. Thus we will be able to draw conclusions about the impact of exposure and experience on the measurement of preferences for innovations in service delivery.

\section{Author affiliations}

${ }^{1}$ Flinders Health Economics Group, School of Medicine, Flinders University, Adelaide, South Australia, Australia

${ }^{2}$ Centre for Health Economics, Monash University, Clayton, Victoria, Australia

${ }^{3}$ School of Psychology, Flinders University, Adelaide, South Australia, Australia

${ }^{4}$ Department of Rehabilitation and Aged Care, School of Health Sciences, Adelaide, South Australia, Australia

${ }^{5}$ Centre for Research in Geriatric Medicine, University of Queensland, Brisbane, Queensland, Australia

${ }^{6}$ School of Nursing and Midwifery, Flinders University, Adelaide, South Australia, Australia

${ }^{7}$ Rehabilitation Studies Unit, Sydney Medical School Northern, University of Sydney, St Leonards, New South Wales, Australia

Acknowledgements The authors are grateful for the in-kind support from these organisations and from other partner organisations including the University of Sydney, Monash University and the University of Queensland.

Contributors JR is the project lead who initiated the idea, led the grant application and drafted the manuscript. JR, ML and EL led the design of the choice experiments. JR and ML led the design of the economic evaluations. ML and JP led the design of the qualitative interviews. All authors had input into the design of the economic evaluations and the design of the discrete choice experiments.

Funding This work was supported through an Australian Research Council Linkage Project, grant number LP110200079. Additional financial contributions were received from the following aged care organisations: ACH Group, Helping Hand, Resthaven, Hammondcare and Catholic Community Services.
Competing interests None.

Ethics approval Flinders University Social and Behvioural Research Ethics Commitee.

Provenance and peer review Not commissioned; peer reviewed for ethical and funding approval prior to submission.

Open Access This is an Open Access article distributed in accordance with the Creative Commons Attribution Non Commercial (CC BY-NC 4.0) license, which permits others to distribute, remix, adapt, build upon this work noncommercially, and license their derivative works on different terms, provided the original work is properly cited and the use is non-commercial. See: http:// creativecommons.org/licenses/by-nc/4.0/

\section{REFERENCES}

1. Australian Government Productivity Commission. Trends in aged care services: some implications. Productivity Commission Research Paper. September 2008.

2. Treasury. Intergenerational Report. Canberra, 2010.

3. Commonwealth of Australia. Living longer. Living better. Canberra: Department of Health and Ageing, 2012.

4. National Health and Hospitals Reform Commission (NHHRC). A healthier future for all Australians: final report of the National Health and Hospitals Reform Commission. June 2009.

5. Aged and Community Services Australia. Consumer Directed Care in Community Care. November 2008.

6. Glendenning C, Challis D, Fernandez JL. Evaluation of the individual budgets pilot programme. Social Policy Research Unit, University of York, 2008.

7. Carlson BL, Foster L, Dale SB, Brown R. Effects of cash and counseling on personal care and well-being. Health Ser Res 2007:42:467-87.

8. Howe A. Is consumer directed care a direction for Australia? Australia: Alzheimers, 2006.

9. Ottmann G, Allen J, Feldman P. Self-directed community aged care for people with complex needs: a literature review. Melbourne: UCCO/Deakin University QRN, 2009.

10. Hardy I. The Vincent Fairfax Churchill Fellowship to investigate "consumer-directed" aged care policy and practice. The Winston Churchill Memorial Trust of Australia, 2008.

11. Ratcliffe J, Laver K, Couzner L, et al. Health economics and geriatrics: challenges and opportunities. Geriatrics, Intech Open Science 209-234.

12. Lanscar E, Louviere J. Conducting discrete choice experiments to inform health care decision making. A user's guide.

Pharmacoeconomics 2008;26:661-7.

13. Ratcliffe J, Buxton M. Patient's preferences regarding the process and outcomes of high technology medicine: an application of conjoint analysis to liver transplantation. Int $J$ Technol Assess Health Care 1999;15:340-51.

14. Mooney G. Key issues in health economics. New York: Harvester Wheatsheaf, 1994.

15. Ezzy D. Qualitative analysis: practice and innovation. NSW: Allen \& Unwin, 2002.

16. Coast J, Al-Janabi $\mathrm{H}$, Sutton $\mathrm{EJ}$, et al. Using qualitative methods for attribute development for discrete choice experiments: issues and recommendations. Health Econ 2012;21:730-41.

17. De Bekker-Grob E, Ryan M, Gerard K. Discrete choice experiments in health economics: a review of the literature. Health Econ 2012;21:145-72.

18. Hensher D, Rose J, Greene W. Applied choice analysis-a primer Cambridge University Press, 2005.

19. Louviere J, Hensher D, Swait J. Stated choice methods: analysis and application. Cambridge University Press, 2000.

20. Burgess L, Street D. The optimal size of choice sets in choice experiments. Statistics 2006;40:507-15.

21. Coast J, Flynn T, Natarajan $L$, et al. Valuing the ICECAP capability index for older people. Soc Sci Med 2008;67:874-82.

22. Euroqol. Measuring self reported health: an international perspective based on EQ-5D. Hungary: Springmed Publishing, 2004.

23. Rosenberg M. Society and the adolescent self-image. Princeton, NJ Princeton University Press, 1965

24. Ranzijn R, Keeves J, Luszcz M, et al. The role of self-perceived usefulness and competence in the self-esteem of elderly adults: confirmatory factor analyses of the Bachman revision of Rosenberg's Self-esteem Scale. J Gerontol Psychol Sci 1998;53B:96-104. 
25. Pearlin L, Schooler C. The structire of coping. J Health Soc Behav 1978;19:2-21.

26. Al-Janabi H, Coast J, Flynn T. What do people value when they provide unpaid care for an older person? A meta-ethnography with interview follow up. Soc Sci Med 2008;67:111-21.

27. Walters $\mathrm{S}$, Brazier $\mathrm{J}$. What is the relationship between the minimally important difference and health state utility values? The case of the SF-6D. Health Qual Life Outcomes 2003;11:1-4.
28. Glick H, Briggs A, Polsky D. Quantifying stochastic uncertainty and presenting results of cost effectiveness analyses. Expert Rev Pharmacoeconomics Outcomes Res 2001:1:25-36.

29. Briggs A, et al. Thinking outside the box: recent advances in the analysis and presentation of uncertainty in cost-effective studies. Annu Rev Public Health 2002;23:377-401. 